\title{
ILCEA
}

Revue de l'Institut des langues et cultures

d'Europe, Amérique, Afrique, Asie et Australie

16 | 2012

La culture progressiste à l'époque de la guerre froide

\section{Gérard Raulet (dir.), Les Romantismes politiques en}

Europe

Éditions de la Maison des sciences de l'homme, coll. « Philia », 2009

Marc Béghin

\section{(2) OpenEdition}

Journals

Édition électronique

URL : http://journals.openedition.org/ilcea/1603

DOI : 10.4000/ilcea.1603

ISSN : 2101-0609

Éditeur

UGA Éditions/Université Grenoble Alpes

Édition imprimée

ISBN : 978-2-84310-232-5

ISSN : 1639-6073

Référence électronique

Marc Béghin, "Gérard Raulet (dir.), Les Romantismes politiques en Europe », ILCEA [En ligne], 16 | 2012, mis en ligne le 04 juillet 2012, consulté le 22 mars 2021. URL : http://journals.openedition.org/ilcea/ 1603 ; DOI : https://doi.org/10.4000/ilcea.1603

Ce document a été généré automatiquement le 22 mars 2021.

(c) ILCEA 


\section{Gérard Raulet (dir.), Les Romantismes politiques en Europe}

Éditions de la Maison des sciences de l'homme, coll. « Philia », 2009

Marc Béghin

\section{RÉFÉRENCE}

Gérard Raulet (dir.), Les Romantismes politiques en Europe, Éditions de la Maison des sciences de l'homme, coll. «Philia », 2009, 625 pages.

1 Avec Silvio Vietta, Karl Heinz Götze, Manfred Frank ou encore Robert Legros, c'est la fine fleur de la recherche en histoire des idées qui émarge au sommaire de ce nouveau titre de l'exigeante collection "Philia", aux Éditions de la Maison des sciences de l'homme. Il faut savoir gré à Gérard Raulet d'avoir pu réunir dans ce volume broché de plus de 600 pages - sans index, hélas ! - qu'orne en couverture un portrait de Lamartine avec, en médaillon, ceux d'Adam Müller, de Chateaubriand et de Frédéric Schlegel, d'aussi prometteuses signatures, mais il convient plus encore de saluer la très bonne tenue d'ensemble de toutes les contributions, soit vingt-huit au total. Elles se répartissent suivant quatre axes: "L'archive romantique du xIx siècle. Révolution, nation, Europe » (p. 11-165), «Le creuset allemand» (p. 169-298), «L'Europe romantique " (p. 299-435) et "Le 'romantisme politique', enjeu idéologique» (p.437-616). Aucune ne dénote sensiblement par rapport à un niveau moyen qu'on n'hésitera pas à qualifier d'élevé, les plus anecdotiques (il en est une sur la famille royale en exil durant la Monarchie de Juillet) offrant une respiration bienvenue, ainsi qu'il convient à un recueil adroitement distribué. Mieux, l'ouvrage constitue à tous égards une somme - ce qui n'est pas un faible mérite pour un sujet d'aussi vaste envergure - et mérite à tous les titres de figurer en bonne place dans la bibliothèque du germaniste, de l'historien, du politologue ou plus largement de l'honnête homme, selon une expression aujourd'hui hors de mode. 
2 Il n'en est pas moins nécessaire d'interroger d'abord certains des partis éditoriaux qui ont présidé à la sélection des contributions. Était-il bien judicieux de faire droit à celles d'entre elles qui ne traitent le sujet que par la négative ou par la bande («L'Europe de Guizot ", "L'Europe selon Cavour ") ? La première citée (Servane Marzin) présente d'ailleurs un phénomène bien connu d'excessive empathie de l'auteur de thèse pour son objet d'étude: Guizot ferait preuve de lucidité sur la question nationale, qui l'oppose aux Romantiques, en voyant qu'elle "porte en elle une passion destructrice qu'il refuse [...] : le nationalisme ». Quant à la seconde (Frédéric Le Moal), on ne peut l'admettre à figurer ici que dans la mesure où Cavour est l'antithèse de Mazzini, qui lui, est bien une personnalité "définitivement [sic] romantique " (Francesco Guida). D'autre part, il est probable qu'on éprouvera quelque difficulté à discerner l'originalité du large panorama proposé par Gilbert Merlio sous le titre-valise «La critique de la civilisation dans le romantisme politique ».

Sur le plan du détail, on relève quand même, qui gâtent à des degrés divers le plaisir de lecture, des naïvetés (y a-t-il lieu de s'offusquer que Thomas Mann, dans sa conférence en forme d'autocorrection "Von deutscher Republik», fasse appel à Novalis qu'il n'aurait découvert qu'en 1920, ou ne cite que six fois Adam Müller?), des anachronismes ("Müller est proche de Norbert Elias»), des pléonasmes (« interprétation herméneutique »), des bévues (les Elemente der Staatskunst sont-ils le « chef-d'œuvre » de Müller, ou simplement son œuvre maîtresse ?), des paresses de traduction (ne s'est-il donc trouvé personne pour aménager l'anglais des pages 366 et 369 ainsi que le poème de Byron à la page 425 ?), des constructions syntaxiques hautement douteuses («tout aussi peu qu'il ne puisse s'extraire de lui-même, il ne peut davantage s'extraire de l'État»), des usages rectionnels parfois hasardeux car issus de l'allemand ("sensibilité pour ", " circonscrit sur ", par exemple), des répétitions peu inspirées («note tenue » trois fois en deux pages) et d'assez vilaines métaphores («le divin a tangenté l'humanité »). À tout cela s'ajoutent aussi des approximations intellectuelles. Le plus petit dénominateur commun que constitue, dans le romantisme politique, « une certaine théologie politique » (sic), peut-il être rabattu sur l'idée «à la mode » (Roques) des racines chrétiennes de l'Europe?

4 Tout cela réservé, le livre est bon, et même très bon, car il bénéficie pleinement du stimulant jeu d'échos et de correspondances qui s'établit en son sein. Là, par exemple, où Christine de Gemeaux, qui livre un condensé clairement organisé de son travail d'habilitation sur Daniel Jenisch et Adam Müller, voit dans les écrits de ce dernier « la magna charta du romantisme politique "-sans toutefois rapporter la preuve de l'influence effective de Müller sur ses pairs en romantisme, hormis le fait que Marx l'a lu « attentivement »-, Karl Heinz Götze, seul à pointer ici le fait tout à fait décisif que manquent encore des études sérieuses sur la question de savoir « de quelle manière et dans quelle mesure l'aristocratie, les États et l'Église ont [...] utilisé les démarches romantiques ", juge "à peine exagéré de dire que si le romantisme n'avait produit que des œuvres de cet acabit, il serait depuis longtemps tombé aux oubliettes ». Un même effet correcteur s'observe au sujet de Carl Schmitt, qui doit à une singulière et troublante fascination française pour son œuvre d'être maintes fois cité sans la distance nécessaire - à l'exception notable de Michael Löwy. Effet de recueil, objecterat-on, que ces redites concernant Schmitt. Reste quand même une difficulté massivement perceptible à rendre raison des contradictions inhérentes au phénomène 
analysé. Ainsi de la coexistence, chez Müller, d'aspects " passéistes et modernes », qui s'expliquerait « par la multiplicité des facettes du romantisme politique »...

5 C'est à ce genre de tautologies qu'échappent seuls - ou paraissent échapper - les quelques auteurs qui poussent l'audace du questionnement jusqu'à révoquer en doute la notion même de romantisme politique, à l'image de Götze posant à titre de thèse que le romantisme ne devient proprement politique qu'à compter du moment où la théorie politique du romantisme allemand «s'abolit en tant que théorie romantique distincte pour s'intégrer sous forme de fragments à l'arsenal argumentatif non seulement du conservatisme allemand, mais aussi à celui du libéralisme ", ou qui, non moins hardiment, en proposent une vision aux contours très nettement arrêtés, tel Lucien Calvié martelant la thèse heinéenne que "l'Allemagne, même en apparence radicalement oppositionnelle, [est] toujours du côté de la contre-révolution ».

Il est dommage enfin que manquent à s'expliquer parfois les auteurs qu'aura quelque peu submergés la richesse de leur thématique (le concept médiéval d'ordo comme "participant d'une conception dynamique du temps ", les implications exactes de cet État chrétien ou "principe catholique » que Müller «réintègre dans la pensée de l'État ", eussent ainsi mérité quelques éclaircissements), ou qu'ait gagné tel autre, dissertant des énigmatiques «förmliche Staatsprozesse » qu'évoque l'essai novalisien sur l'Europe, la tentation de s'exprimer en un style totalement ésotérique : qu'est-ce que la « dynamique du schize » ou une « saillance ponctuelle»?

Beaucoup plus louable apparaîtra sans doute l'effort de plusieurs contributeurs pour mettre en relation l'objet de leur réflexion avec notre actualité. Gérard Raulet note ainsi que la «bévue» de Schlegel, lecteur du traité de paix kantien, sur la notion moderne de républicanisme sacrifiée à celle de cosmopolitisme, induit aujourd'hui « les apories de la 'mondialisation' tiraillée entre des principes républicains auxquels on ne cesse d'objecter le respect des différences communautaires ou nationales et l'abandon de ces mêmes principes au nom d'une pseudo-démocratie mondiale ». Robert Legros renvoie dos à dos, dans un texte à haute teneur axiologique, pensée libérale et pensée romantique pour en arriver à définir normativement la nation comme un «espace commun et public où toutes les arrogances individuelles et communautaires puissent être suspendues, afin que soit préservée la dignité de l'homme comme tel ». Christian Roques fait observer que la philosophie thomiste de la guerre juste, à laquelle fait retour Adam Müller, a également inspiré la mouvance conservatrice bushienne dans son effort pour légitimer la guerre en Irak. Christine de Gemeaux explique ce qu'il en est pour Müller de la «circulation des échanges [intellectuels]» au sein de l'Étatmédiateur, « qui lui inspire l'idée d'une raison langagière » et fait de lui l'anticipateur d'une "pensée à la Habermas, d'un 'agir communicationnel', qui ne serait viable qu'à travers la médiation de l'État ». Jeffrey Reid, enfin, professeur agrégé de philosophie à l'université d'Ottawa, porte l'attention sur la critique hégélienne du romantisme et spécialement de l'ironie romantique, sous les trois formes qu'incarnent Novalis (hypertrophie $\mathrm{du}$ sentiment), Schlegel (hypertrophie de l'entendement) et Schleiermacher (" hybride monstrueux de chacune des deux formes ironiques »), et sur ce que cette critique en termes de barbarie des "unilatéralités de l'intuition et de l'entendement » peut vouloir dire pour nous, postmodernes. Ce ne sont là que quelques exemples, choisis parmi les plus significatifs, des nombreuses incitations que contient ce très solide et très riche volume à prolonger la réflexion sur le romantisme politique et la fascination qu'il continue d'exercer aujourd'hui. 